\title{
Neurohumoral Pathway in Heart Failure
}

\author{
Dyah Wulan Anggrahini \\ Department of Cardiology and Vascular Medicine, Faculty of Medicine, Public Health and Nursing, Universitas Gadjah \\ Mada - Dr. Sardjito General Hospital, Yogyakarta, Indonesia
}

Corresponding author:

Dyah Wulan Anggrahini, MD, - email: wulan.anggrahini@gmail.com

Department of Cardiology and Vascular Medicine, Faculty of Medicine, Public Health and Nursing, Universitas Gadjah Mada - Dr. Sardjito Hospital

Jalan Farmako Sekip Utara, Yogyakarta, Indonesia 55281

\section{ABSTRACT}

Heart Failure is now considered as one of the leading cause for mortality and morbidity. It is affecting several organs and cause organ damages due to the myocardial failure to pump inadequate oxygenated blood to the body including metabolites, to end organs and peripheral tissues. Heart failure results from multifactorial mechanism including neurohumoral activations including increased activity of the sympathetic nervous system, renin-angiotensin aldosteron system, vasopression and the atrial natriuretic peptide. This neurohumoral pathway has significant contribution to the development of myocardial dysfunction that lead to clinical manifestation of heart failure. Some of the markers in these pathways have now been considered as an independent predictors of prognosis in heart failure patient.

\section{Introduction}

Heart failure is a syndrome that currently being understood as a systemic failure that result in decrease oxygen perfusion due to dysfunction of myocardium in performing good contractility. The worldwide incidence of heart failure has increased to 26 millions, and $74 \%$ of the cases cause one comorbidity that worsens patient health status. In Asia, the prevalence is ranging from $1-5 \%$ and overall prevalence is estimated to be 4.2 million in China and 1.3-4.6 million in India ${ }^{1,2}$.

The failure of ventricular function causes inability of the heart to deliver adequate blood to the body to meet end-organ metabolic demands and oxygenation at rest or during mild exercise will further result in organ damages. The failing heart strives to balance "preload" and "afterload" for compensation of impaired contractility and to deter the development of congestion, which occur via several interdependence mechanisms.
Regulation of circulating blood volume in physiological condition is controlled to maintain the cardiovascular homeostasis. The damage in structure and function of the myocardium lead to changes in these regulations and result in compensatory mechanism that is regulated through the activation of neurohumoral and autonomic nervous system. The disruption in receptor activation cause imbalance in the autonomic system with increased sympathetic activity and diminished vagal reflexes, both of which may have profound effects on cardiac function and structured. Furthermore, the decreased ventricular function and activation of the SNS will cause low blood perfusion to the kidney and stimulates the activation of renal aldosterone angiotensin system and vasopressin release ${ }^{3,4}$. The activation in these complex neurohumoral mechanisms support and maintain tissue organ perfusion. The schematic neurohumoral adaptation in heart failure is shown in figure 1 and the processes by which depressed 
myocardium may lead to systemic figure 2 . decompensation are shown in the

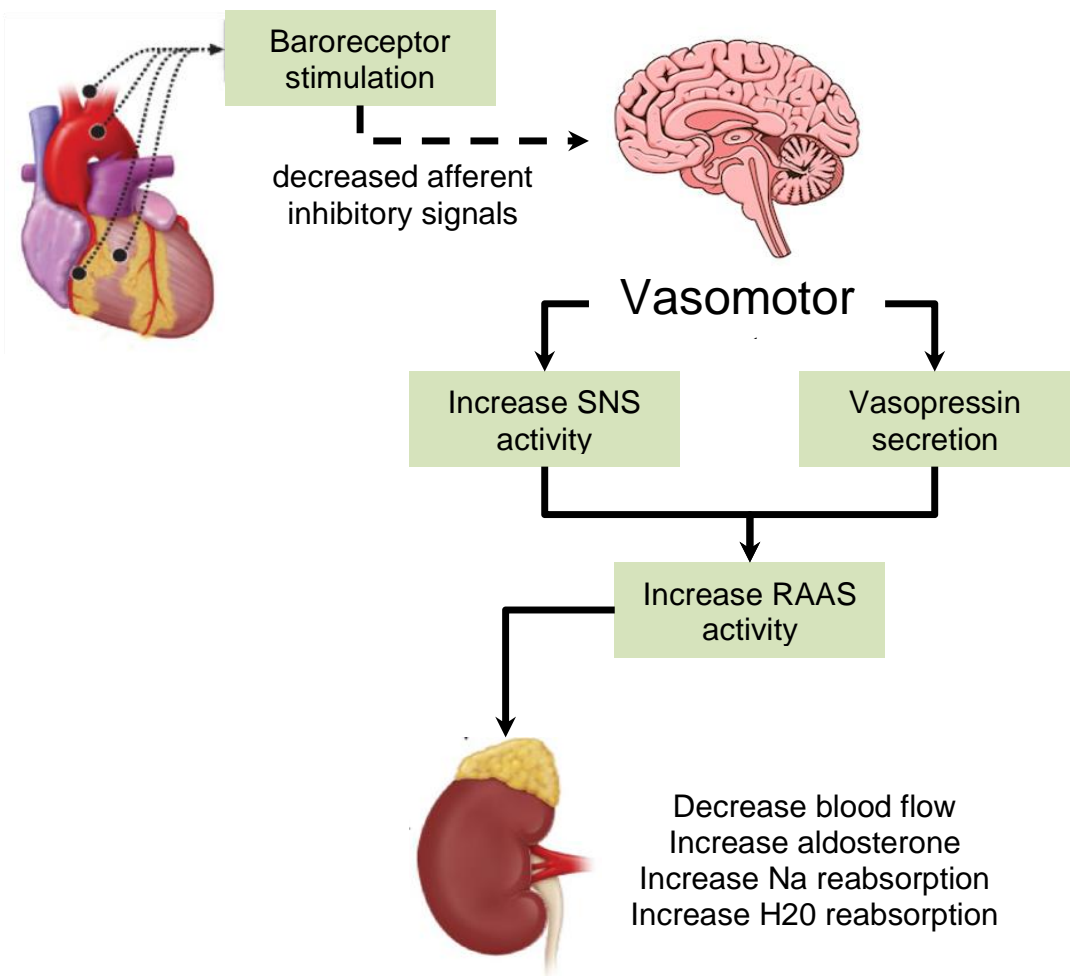

Figure 1. The activation of SNS and Neurohumoral system in the failing heart (reproduced from Hartupee $\mathrm{J}$ and Mann DL, 2017)

Figure 2. The process of heart failure leads to end stage organ dysfunction

Activation of sympathetic nervous system pathways

In healthy individuals, the balance in sympathetic and

\section{End-stage \\ multi \\ organ \\ failure}


body changes, the baroreceptor in the aortic arch and carotid sinus, as well as mechanoreceptors at the cardiorespiratory system senses arterial wall tension and produce signals ${ }^{3}$. These signals cause increase in sympathetic impulse through norepinephrine or parasympathetic one via acetylcholine. At the peripheral vessels, the chemoreceptors and metabolic receptors in the muscles will sense acid-base balance and oxygenation of the blood and further produce stimulation in the sympathetic impulse. The changes in mechanical and biochemical condition like hypoxia, hypotension or acid-base imbalance will be transmitted and sensed by those baroreceptors creating a feedback mechanism to maintain the cardiovascular homeostasis ${ }^{3}$.

During the early course of heart failure, the activation of the Sympathetic Nervous System (SNS) is one of the most important adaptations that occur in the very early stage. The failing ventricular function leads to increase in SNS signaling with subsequent release and reduce uptake in adrenergic neurotransmitter Norepinephine (NE) by the nerve endings, causing increased in circulating level of this neurotransmitter. In response, the parasympathetic receptor activity become dysfunctional by these sympathetic simulation, which in turn lead to increase in systemic vascular resistance and heart rate ${ }^{3}$.

\section{The renin-angiotensin-}

\section{aldosteron system (RAAS)}

pathways

The renin angiotensin aldosterone system functions as a controller in intravascula volume and resistance.
During physiological condition, the RAAS is main hormonal signaling cascade to maintain body fluid and blood pressure homeostasis ${ }^{5}$. RAS influences BP by regulating sald and water balance and vasoconstriction. In addition, in the in vitro and in vivo model, the RAAS has plaucible function in the cellular and tissue remodeling as well as the increase response in inflamatory process. Thus, the inapporpriate response of the RAAS during certain pathological condition will lead to tissue remodeling and dysfunction in cardiovascular system ${ }^{6}$.

In heart failure syndrome, the failed contractility of the ventricel causes renal hypoperfusion and together with sympathetic activity, the RAAS is activated in the later stage aafter SNS activation. This system is very sensitive and is activated with the extrication of renin from juxtaglomerular apparatus. Renin is first secreted, and it is a hormone that mediates the conversion of angiotensin I ro angiotensine II by angiotensin-converting enzyme (ACE). Reports showed that during heart failure, the activity of ACE is increased as shown that the tissue expression increased in the myocardium. This further supported with the increased in Angiotensin II activity during heart failure that stimulates vasoconstriction, cellular growth and tissue remodeling, extracellular matrix synthesis which may exacerbate the fibrosis in heart failure condition ${ }^{3,6}$. There are two opposing receptors that binds to Angiotensin II and mediates its activity, Angiotensin recepor type 1 and Angiotensin receptor type 2 . The activation of type 1 receptor leads to cell growth, vasoconstriction and mediates fibrotic process. The activation of type 2 receptors leads to 
the inhibition of cell growth and vasodilatation. Indeed, the effects of Angiotensin II on cardiovascular, renal, and cerebral functions are mediated through the activation of angiotensin type 1 (AT1) receptors, but these actions are counteracted by activation of the AT2 receptor ${ }^{3}$. Aldosterone has similar actions with unfavorable effects of angiotensin II. Aldosterone provokes hypertrophy and fibrosis within the vasculature and myocardium, resulting in ventricular stiffness, endothelial cell and baroreceptor dysfunction, and the inhibition of norepinephrine uptake ${ }^{6}$.

\section{Vasopressin}

The arginin vasopressin system plays role in the mechanism of heart failure through its action in mediating the water clearance in the kidney. The release of vasopressin is provoked by Angiotensin II after it stimulates the thirst center in the brain. Normally in the setting of increased osmolality AVP is released resulting in increased water retention, which returns osmolality to its normal physiological set point. However, AVP levels are inappropriately elevated in many patients with heart failure ${ }^{3}$.

\section{The natriuretic peptide}

Recently, the natriuretic peptide hormon has been reported to play its role in the compensatory mechanism of heart failure. Previously, Brain Natriuretic Peptide (BNP) has been widely reported and been used as a biomarker for heart failure. It has prognostic value in patients with post myocardial infarction and associated with decreased survival in those with reduced LVEF. The Natriuretic Peptide (NP) is a cardiac hormone reported being released in myocardium and the endothelium, with pleiotropic cardiovascular and metabolic properties. The wall stress due to volume overload or pressure overload during heart failure induces synthesis of natriuretic peptide, thus there is increased secretion of NP to counterbalance the increase effect of renin-angiotensin-aldosterone

system $^{7,8}$.

The release of NP in heart failure will induces vasodilation, natriuresis, and supress the RAAS. In the kidney, this hormone increases glomerular filtration rate by increasing afferent arteriolar dilation in addition to efferent arteriolar constriction, while in the different level of the nephron ANP inhibits water and sodium reabsorption. Moreover, NPs has effect on antagonizing cardiac hypertrophy and fibrosis leading to myocardial remodeling in heart failure. The complete pleiotropic effect is shown in figure $3^{9}$. The clearance of NPs is mediated through a specific natriuretic peptide degrading receptor called NPR-C that binds the NPs and through a mechanism of endocytosis it degraged and forms an inactive molecule (Figure 4$)^{8}$. A breakthrough discovery in fdrug development recently has targeted the inhibition of NPs as one mechanism in the treatment approach of heart failure ${ }^{10}$. 
Inactive NP fragments

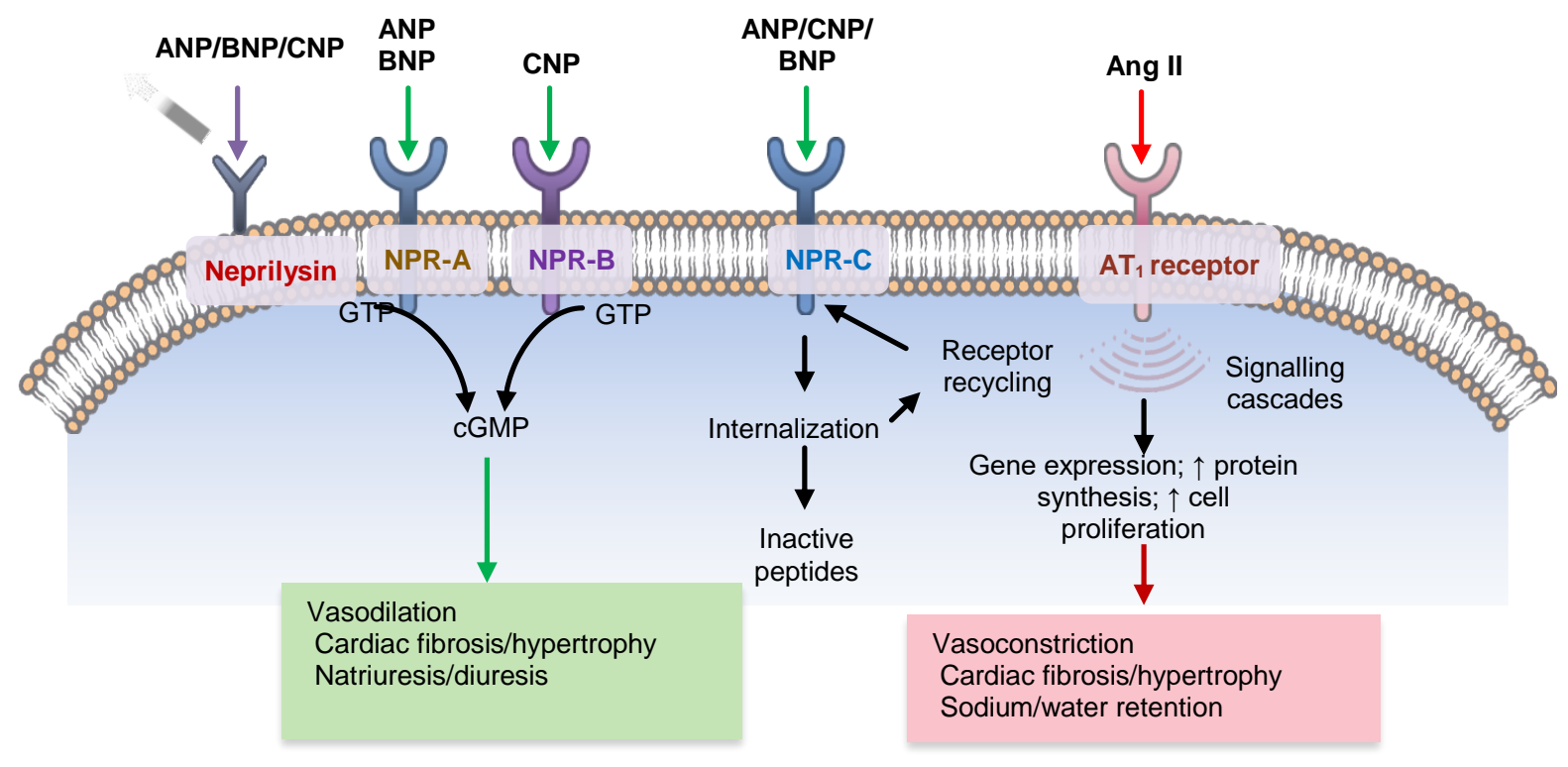

Figure 3. Effects of the natriuretic peptide system: cardiovascular and renal effects of NP system counteract the action of RAAS

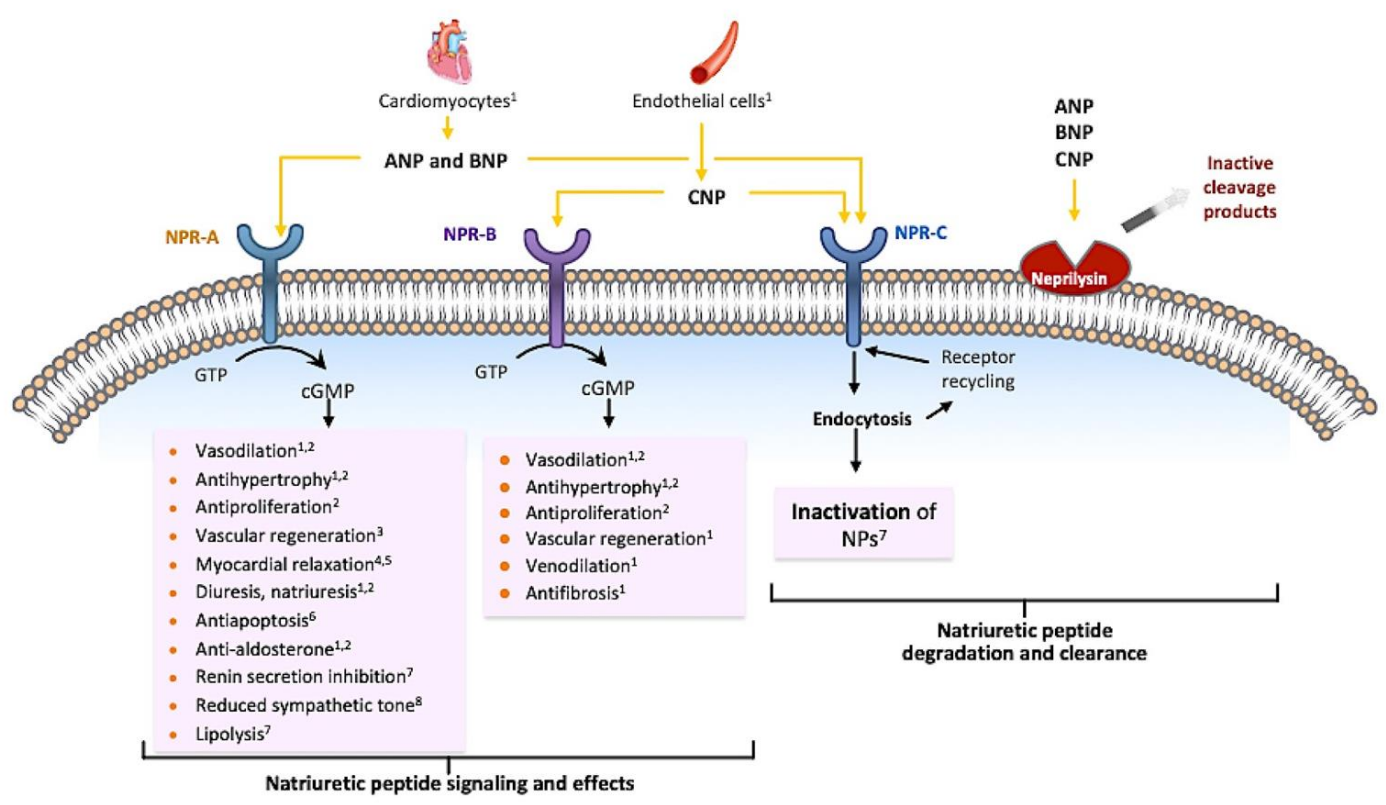

Figure 4. Natriuretic peptides signaling and the degradation and clearance

\section{References}

1. Lam C.S., Teng T.K., Tay W.T., Anand I., Zhang S., Shimizu W., et al. 2016. Regional and ethnic differences among patients with heart failure in Asia: the Asian sudden cardiac death in heart failure registry. Eur Heart J, 37:3141-3153.

2. Dewan P., Jhund P.S., Shen L., Petrie M.C., Abraham W.T., Atif Ali M., et al. 2019. Heart failure with reduced ejection fraction: comparison of patient characteristics and clinical outcomes within Asia and between Asia, Europe and the 
Americas. Eur J Heart Fail, 21:577-587.

3. Hartupee J., Mann D.L. 2017. Neurohormonal activation in heart failure with reduced ejection fraction. Nat Rev Cardiol, 14: 30-38.

4. Angelis E.D., Pcoraro M., Rusciano M.R., Ciccarelli M., Popolo A. 2019. Cross-Talk between neurohormonal pathways and the immune system in heart failure: a review of the literature. Int $\mathrm{J} \mathrm{Mol} \mathrm{Sci,}$ 20:1698.

5. Sullivan R.D., Mehta R.M., Tripathi R., Reed G.L., Gladysheva I.P., et.al. 2019. Renin activity in heart failure with reduced systolic functionnew insight. Int $\mathrm{J}$ Mol Sci, $28 ; 20$.

6. Orsborne C., Chaggar P.S., Shaw S.M., Williams S.G. 2017. The renin-angiotensinaldosterone system in heart failure for the non-specialist: the past, the present and the future. Postgrad Med J, 93:29-

37.

7. Maisel AS, Duran JM, Wettersten

$\mathrm{N}$.

Natriuretic peptides in heart failure: atrial and

$B$ type natriuretic peptides. 2018. Heart Fail Clin, 14:13-25

8. Potter LR. 2011. Natriuretic peptide metabolism, clearance and degradation FEBS J, 278:1808-1817.

9. Dewan P., Docherty K.F., McMurray J.J.V. 2019. Sacubitril/Valsartan in Asian patients with heart failure with reduced ejection fraction. Korean Circ J, 49:469 484.

10. Mangiafico S., CostelloBoerrigter L.C., Andersen I.A., Cataliotti A., Burnett J.C.Jr. 2013. Neutral endopeptidase inhibition and the natriuretic peptide system: an evolving strategy in cardiovascular therapeutics. Eur Heart J, 34:886-893. 